\section{Anna Paul}

Gustav Dobos

\title{
Selbstfürsorge für Patienten und ihre Therapeuten: 10 Jahre Mind-Body Medicine Summer School in Essen
}

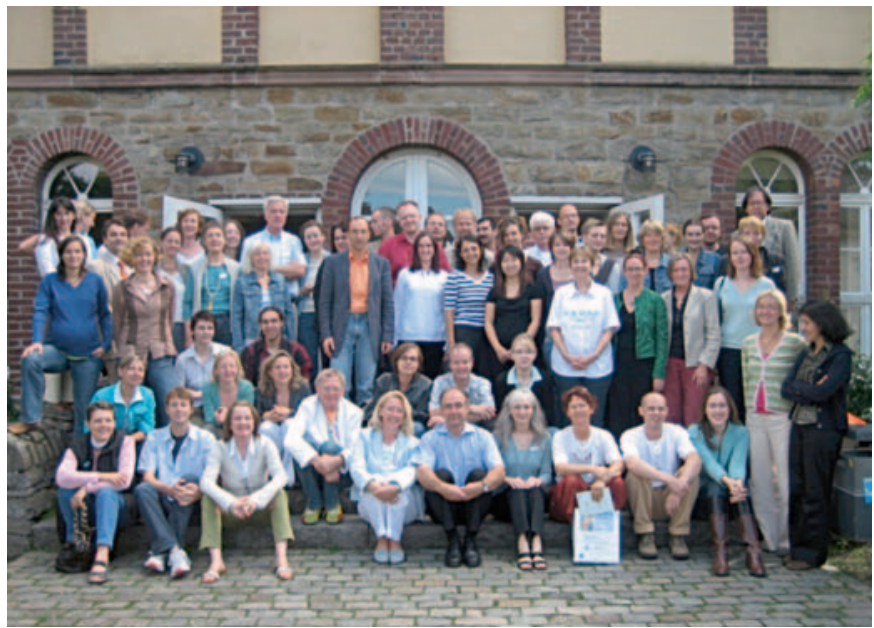

Abb. 1. Die Teilnehmer der 1. Mind-Body Medicine Summer School 2006.

nes der momentan in Deutschland

Berufung etwas anderes vorgestellt haben als ein Leben zwischen Rezeptblock und einer 5-Minuten-Medizin. Sie suchen nach einem besseren Verständnis von dem, was Krankheit und Gesundheit ausmacht, und sehnen sich nach Inspiration und Anregungen. Viele möchten sich gezielt mit gleichgesinnten Kolleginnen und Kollegen vernetzen, um nachhaltig etwas in ihrem Berufsalltag, aber auch in ihrem Leben verändern zu können.

Was ist die Mind-Body-Medizin? Selbst das zu praktizieren, was man den Patienten mitgeben will - das ist vielleicht der entscheidendste Unterschied zu anderen Bereichen der Medizin, meint Nils Altner. Der Bildungsund Gesundheitswissenschaftler, der regelmässig Workshops auf der Summer School abhält, gehörte vor rund einem Dutzend Jahren zu den ersten Achtsamkeitstherapeuten Deutschlands - heute ist deren Zahl auf über 1000 angewachsen. MBSR, die «mindfulness-based stress reduction», ist eibekanntesten Programme der MindBody-Medizin: Sie basiert auf jener «Achtsamkeit», die der amerikanische Molekularbiologe und Stressforscher Jon Kabat-Zinn aus den Dimensionen asiatischer Weisheit entlieh und in die säkulare Welt übertrug. Kern seines erfolgreichen Programms ist die Stressbewältigung durch eine gezielte Lenkung von Aufmerksamkeit.

«Mindfulness» - fokussierte Wahrnehmung in kürzeren oder längeren Konzentrationsübungen oder auch Meditationen - will nicht nur kurzfristig Einfluss auf den Körper nehmen, wie das etwa bei einer Entspannungsübung der Fall wäre. Ein Achtsamkeitstraining hat das Ziel, zu einer nachhaltig gesundheitsfördernden Lebenshaltung im Alltag zu führen. Ärzte und Therapeuten können diese Entwicklung anstossen, einüben und ihr zu einer Stabilisierung verhelfen - daran arbeiten müssen die Patienten selbst. Der Essener Internist Ulrich Deuse erklärt sorgung sind, weil sie sich unter ihrer

\section{KARGER}

(c) 2015 S. Karger GmbH, Freiburg
Dr. rer. medic. Anna Paul

Klinik für Naturheilkunde und Integrative Medizin, Kliniken Essen-Mitte

Medizinische Fakultät, Universität Duisburg-Essen, Knappschafts-Krankenhaus

Am Deimelsberg 34a, 45276 Essen, Deutschland

a.paul@kliniken-essen-mitte.de 
regelmässig auf der Summer School ärztlichen Kolleginnen und Kollegen, wie er dieses Konzept in seine Arztpraxis geholt hat und welche positiven Erfahrungen er damit gemacht hat.

Vor allem die jungen Ärzte und Medizinstudenten, die zur «Mind-Body Medicine Summer School» nach Essen kommen, interessieren sich für Praktiken wie die Achtsamkeit nicht nur, um ihre therapeutischen Fähigkeiten zu verbessern. Sie erkennen darin auch ein wichtiges Mittel zum Selbstschutz - vor seelischer und körperlicher Überlastung. Dass sich die junge Medizinergeneration nicht mehr so auspowern möchte wie ihre Vorgänger, ist einer der Gründe, warum studentische Initiativen wie «Medizin und Menschlichkeit (MUM)» oder «Einherz» entstanden sind. Den angehenden Ärztinnen und Ärzten geht es darum, die Beziehung zwischen Arzt und Patient wieder verstärkt in den Mittelpunkt zu stellen. Gemeinsam mit älteren, erfahreneren Kollegen entstehen auf der Summer School interessante "Tandems» von noch unbelasteten Visionen und langjährigen Erfahrungen, die in keinem Lehrbuch stehen.

Was ist das Neue an der Mind-Body-Medizin? «Die Ordnungstherapie der Naturheilkunde war sehr stark auf die Empfehlungen des Pfarrers Kneipp ausgerichtet», sagt Andreas Michalsen, ehemaliger Oberarzt der Essener Klinik und heute Professor für klinische Naturheilkunde an der Berliner Charité. «Das war alles etwas altmodisch, im Ton von Ermahnungen und Ermutigungen. Die Mind-Body-Medizin ist nicht nur eine Erweiterung dieser klassischen Ordnungstherapie, sondern konkreter, gut wissenschaftlich untersucht und global orientiert.»

In Michalsens Abteilung für Naturheilkunde im Immanuel-Krankenhaus in Berlin gehört die Mind-Body-Medizin zum Repertoire jeder Behandlung und wird - wie auch in Essen - von den körperzentrierten Anwendungen der Naturheilkunde ideal unterstützt. «Die Nachfrage von Patientenseite ist gross - wir kommen mit dem Ausbau der
Abteilung kaum hinterher», sagt Michalsen. «Demnächst haben wir 60 Betten auf der Station. Es ist nicht einfach, dafür genügend gut ausgebildetes Personal zu finden - in der Ordnungstherapie wie in der medizinischen $\mathrm{Na}-$ turheilkunde.»

Michalsen wünscht sich, dass noch mehr seiner ärztlichen Kollegen das Potenzial der Mind-Body-Medizin wahrnähmen und realisierten. Eine solche Entwicklung scheint sich gerade im Bereich der Onkologie anzubahnen - die immer mehr zur «integrativen» Onkologie wird, also zu einer Tumormedizin, welche die Möglichkeiten der Naturheilkunde zur Behandlung von Nebenwirkungen der Therapie nutzt. Immer mehr Kliniken, vor allem Universitätskliniken, bieten integrativ-onkologische Therapien an, neben Berlin und Essen z.B. auch das Hamburger Universitätsklinikum Eppendorf.

Eine Arbeitsgruppe vom Universitären Cancer Center und der MartiniKlinik bietet dort Prostatakrebs-Patienten mit hohem Rezidivrisiko zusätzlich zur Operation ein ambulantes 10-Wochen-Programm mit speziellen Übungen und Empfehlungen in den Bereichen Ernährung, Bewegung und Entspannung an. Die Herausforderung besteht darin, das Gesundheitsverhalten der sonst schwer zu erreichenden Männer zu verändern, auch wenn das Erfolg verspricht: «Epidemiologische Studien ergeben Hinweise», sagt der Hamburger Onkologe Matthias Rostock, «dass sich die Mortalität deutlich senken lässt.»

Rostock ist auch in der Schweiz tätig und arbeitet dort mit Claudia Witt zusammen, die - ehemals Charité Berlin - 2014 eine Professur für Komplementäre und Integrative Medizin an der Universität Zürich angenommen hat. "Wir setzen auf die Integrative Onkologie und dabei sehr stark auf die Mind-Body-Medizin», sagt Witt, «als ein Verfahren, das sich gut mit konventionellen Therapien kombinieren lässt. Dabei bieten wir sowohl Einzeltermine als auch Gruppen an.» Zwar sei die Mind-Body-Medizin ein relativ neues Thema in der Schweiz, aber das Interesse von Kolleginnen und Kollegen sei rasch gewachsen. «Wir kooperieren eng mit der Universität Duisburg-Essen und bekommen viel Unterstützung beim Aufbau.»

Claudia Witt hält die Mind-BodyMedizin für ein wunderbares Beispiel für zwei sehr positive Entwicklungen in der Medizin: die zunehmende Wertschätzung interprofessioneller Zusammenarbeit und das Empowerment der Patientinnen und Patienten zu mehr «Self-Care». Das Besondere der Mind-Body-Medizin in Deutschland und der Schweiz, sagt die Ärztin und Epidemiologin, sei, dass naturheilkundliche Selbsthilfestrategien in das Gesamtkonzept integriert wurden.

Der Ursprung der Mind-BodyMedizin nämlich liegt in den USA, wo die Naturheilkunde europäischer Prägung so gut wie keine Rolle spielt. Als die Essener Klinik für Naturheilkunde und Integrative Medizin 1999 gegründet wurde - damals noch als Modellvorhaben des Landes NordrheinWestfalen -, fehlten die geforderten wissenschaftlichen Studien und Belege der Ordnungstherapie. Also suchte das Team in den USA und wurde fündig: in Boston, am Benson-Henry Institute for Mind-Body Medicine.

Dort steht die «Wiege» der internationalen Lebensstilmedizin, die stark von der Stressforschung geprägt ist. Ihr Pionier ist der Kardiologe Herbert Benson. Viele der Krankheiten der westlichen Welt, so seine Ausgangsthese, werden durch Stress ausgelöst oder verschlimmert. Es müsse doch möglich sein, so seine Annahme, die Negativ-Kaskade der Botenstoffe, die dabei im Spiel ist, gezielt «umzudrehen». Analog zur Stressantwort des Organismus nannte Benson diesen Vorgang «Relaxation Response» Entspannungsantwort.

Der Essener Lehrstuhl arbeitet bis heute eng mit dem Benson-Henry Institute zusammen, dessen Mitarbeiter und Schüler auf der Summer School ihre jüngsten Ergebnisse vorstellen. Beispielsweise Eva Selhub, die auf Ben- 


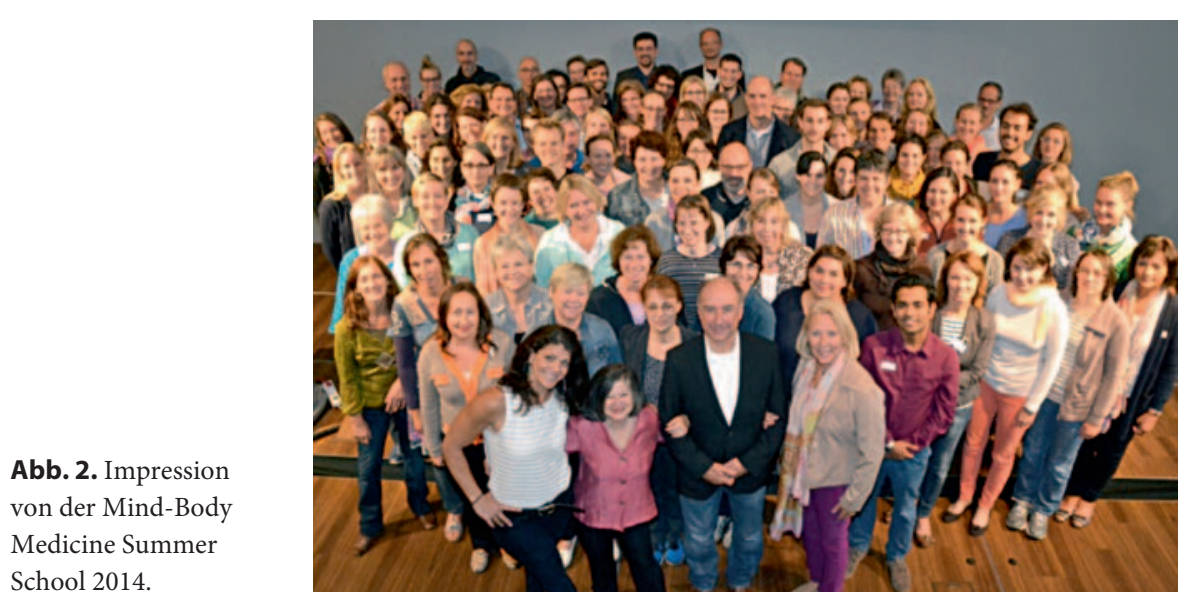

sons Ergebnissen aufbauend eine Theorie der «Love Response» aufbaute und regelmässig auf der Summer School in Essen von ihrer Arbeit mit Patienten berichtet. «Die Forschung macht grosse Fortschritte», bestätigt auch Peg Baim vom Benson-Henry Institute. «Der Zusammenhang zwischen nervlicher Belastung und entzündlichen Erkrankungen wird immer deutlicher. Dank Genforschung und bildgebenden Verfahren lässt sich nun in vielen Bereichen der Medizin - etwa der Psychiatrie, Gastroenterologie oder Onkologie - die zerstörerische Wirkung von Stress nachweisen. Umgekehrt lernen wir, was wir von der MindBody-Medizin und einem gesunden Lebensstil profitieren können.»

Wie funktioniert das? Die MindBody-Medizin konzentriert sich mit einem breiten Spektrum von Interventionen auf die Ressourcen von Patienten - «zum ersten Mal wird die Psyche nicht unter pathogenen Gesichtspunkten gesehen, sondern unter salutogenetischen, mit dem Ziel der Resilienzförderung», sagt Nils Altner. Durch die Anregung und Einübung kognitiver Prozesse und Verhaltensmodifikationen unterstützt sie die Fähigkeiten des Organismus zur Selbstregulation und Selbstheilung. Sie verbessert die Selbstwahrnehmung und Selbstfürsorge und greift auch spirituelle Bedürfnisse auf.

In der therapeutischen Praxis greift die Mind-Body-Medizin auf Elemente traditioneller Heilverfahren (Yoga, Tai Chi, Qigong oder Meditation) genauso zurück wie auf moderne Entspannungsverfahren und Bewusstseinstechniken (wie die bereits zitierte Achtsamkeit, aber auch Progressive Muskelentspannung, Imagination oder Kognitive Verhaltensänderung). Zudem werden Ernährung, Bewegung und soziale Unterstützung als zentrale Aspekte einer gesundheitsfördernden Lebensstilgestaltung gesehen. «MindBody-Medizin bedeutet, dass wir den Patienten in den Mittelpunkt unserer Aufmerksamkeit stellen, um das gesamte Spektrum körperlicher, mentaler, emotionaler, sozialer und spiritueller Einflussfaktoren zu berücksichtigen, das Einfluss auf seine Gesundheit hat», fasst der Institutsgründer Herbert Benson zusammen. «Wichtig ist, dass die Mind-Body-Medizin ein ganzheitlicher, ressourcenorientierter Ansatz bleibt und nicht in Einzelteile zerlegt wird», betont Andreas Michalsen.

Herbert Benson hat mit seinem Team auch ein eigenes Mind-BodyProgramm für Tumorpatienten entwickelt, das auch Grundlage der Arbeit der Abteilung für Integrative Onkologie an den Kliniken Essen-Mitte ist. Einer der prominentesten Schüler von Benson, der Lifestyle-Mediziner Dean Ornish, konnte unter anderem zeigen, dass ein gesunder Lebensstil die Art und Weise verändert, wie Gene im Körper aktiv sind bzw. an- oder abgeschaltet werden. Das kann die Entstehung von Krebs beeinflussen, wie Susan Lutgendorf, Psychoneuroimmunologin an der Universität von Iowa, 2014 auf der Essener Summer School (Abb. 2) zeigte.
Die Mind-Body-Medizin wirkt als präventiver und gesundheitsfördernder Ansatz in immer mehr Bereiche hinein: Nancy Haradzuk, Assistenzprofessorin an der Georgetown University of Medicine in Washington, hat ein MBM-Netzwerk für Studierende aufgebaut. Nils Altner hat einerseits Konzepte für Kindergärten entwickelt, ist aber gemeinsam mit einem Team der Essener Klinik auch am anderen Ende der Altersskala tätig: in der betrieblichen Prävention. Die spezielle Aufgabe besteht angesichts der demographischen Entwicklung darin, Spannkraft und Kreativität bei älteren Arbeitnehmern zu fördern, da diese immer länger aktiv bleiben müssen.

Tobias Esch, Mediziner und Professor an der Hochschule Coburg, hat dort den ersten deutschen Studiengang zur Integrativen Gesundheitsförderung aufgebaut. Esch, der seine berufliche Laufbahn in Essen begann und von dort als Post-Doc zu Herbert Benson an die Harvard Medical School wechselte, hat die Entwicklung der Essener Mind-Body-Medizin von Anfang an begleitet, arbeitet heute unter anderem als Gastprofessor an der Harvard Medical School. Ihn interessieren besonders die kognitiven Aspekte der Mind-Body-Medizin. Er verbindet sie auch mit der Glücksforschung der Positiven Psychologie. «Mich interessieren vor allem die Mechanismen der Belohnung im Gehirn, etwa der Dopaminhaushalt», sagt Esch, «denn eines ist klar: Verhaltensänderungen funktionieren nur, wenn sie sich auch langfristig gut anfühlen.»

\section{Weiterführende Informationen}

- Ein kurzer Film zur Mind-Body Medicine Summer School 2013 steht unter folgendem Link zur Verfügung: https://www.youtube. com/watch?v=JAhSiT9dNaE.

- Eine Literaturliste zum Thema Mind-Body-Medizin ist unter $w w w$. mindbodymedizin.de (http://www. nhk-fortbildungen.de/files/mindbodymedicine_literatur.pdf) verfügbar. 